\title{
МОДЕЛИРОВАНИЕ ХАРАКТЕРИСТИК ЗАЩИТНЫХ ДИЭЛЕКТРИЧЕСКИХ ПОКРЫТИЙ
}

В данной статье представлено то как могут располагаться резонансные частоты диэлектрических слоёв. Решение этой задачи позволит ответить и на другой важный вопрос: как могут располагаться частотные зоны поглощения (а следовательно и отражения) и какова их ширина. В предлагаемой статье даётся решение сформулированной выше задачи для простейшего интерференционного покрытия и следствия решения.

Как известно [1] мощным средством защиты телекоммуникационной аппаратуры являются защитные радиомаскировочные покрытия. Для уменышения эффективной площади рассеяния объектов телекоммуникаций широко применяются покрытия интерференционного типа [1]. Центральная частота области поглощения электромагнитной энергии определяется резонансной частотой самого покрытия, то есть диэлектрическим слоем. При резонансе поверхностное сопротивление резистивной плёнки трансформируется диэлектрическим слоем в волновое сопротивление свободного пространства. В результате электромагнитная энергия поглощается в резистивной плёнке покрытия. Поскольку диэлектрический слой является многорезонансной системой, то и интерференционное покрытие имеет бесконечное число областей поглощения и отражения. Физически ясно, что для расширения частотной области поглощения желательно иметь диэлектрический слой, резонансные частоты которого близко расположены друг к другу. Тогда из-за наличия резистивной плёнки и собственных диэлектрических потерь амплитудно-частотные характеристики соседних резонансов будут перекрываться. Это и обеспечит расширение рабочего диапазона интерференционных покрытий. Здесь возникает вопрос: как в принципе могут располагаться резонансные частоты диэлектрических слоёв? Решение этой задачи позволит ответить и на другой важный вопрос: как могут располагаться частотные зоны поглощения (а следовательно и отражения) и какова их ширина.

В предлагаемой статье даётся решение сформулированной выше задачи для простейшего интерференционного покрытия, состоящего из нерегулярного диэлектрического слоя, в начале которого расположена резистивная плёнка, а в конце помещён электрический экран, имитирующий металлическую поверхность телекоммуникационной аппаратуры. Предполагается, что диэлектрическая и магнитная проницаемости могут меняться по толщине покрытия и волна падает на покрытие под углом девяносто градусов (нормальное падение).

(ㄱ I. Р. Пархомей, В. А. Паюн, Д. О. Сопильняк 
Поскольку диэлектрический слой с электрическим экраном является распределённой цепью, то его поверхностный импеданс является мероморфной функцией относительно комплексной частотной переменной $p[2,4]$ :

$$
Z=\sum_{k=1}^{\infty} \frac{\beta_{k} p}{p^{2}+\omega_{k}^{2}}
$$

где $\beta_{k}$ - положительные числа; $\omega_{k}$ - полюсы поверхностного сопротивления.

Рассмотрим цепь, состоящую из n (чётное число) линий с постоянным волновым сопротивлением (стержневую цепь Ричардса), замкнутую на конце. Потребуем, чтобы первые $\mathrm{n} / 2$ полюсов сопротивления этой цепи были равны $\omega_{k}, \kappa$ $=1,2, \ldots, / 2$. Тогда входное сопротивление цепи Ричардса

$$
\begin{gathered}
Z_{n}(\lambda)=\sum_{k=1}^{n / 2} \frac{\alpha_{k} \lambda}{\lambda^{2}+\zeta^{2}}, \alpha_{k}>0, \\
\lambda=\operatorname{th} p \frac{t_{3}}{n}, \zeta_{k}=\operatorname{tg} \omega_{k} \frac{t_{3}}{n}, t_{3}<\frac{n \pi}{2 \omega_{n / 2}} .
\end{gathered}
$$

Из последних соотношений следует, что $\zeta_{k}>\zeta_{k-1}$.

Теперь найдём выражение для $\alpha_{k}$, при котором Res $Z=\operatorname{res} Z_{n}(\lambda), \mathrm{p}=\mathrm{I} \omega_{k}$, $\mathrm{k} \leq \mathrm{n} / 2$. Для этого обозначим

$$
\begin{aligned}
& Z_{n}(\lambda)=\frac{A_{n}(\lambda)}{B_{n}(\lambda)}, \text { тогда } \\
& \alpha_{k}=2 \frac{A_{n}\left(\lambda_{k}\right)}{\frac{d B_{n}(\lambda)}{d \lambda}}, \lambda=\lambda_{k}=i \varsigma_{k} \text { учитывая, что } \frac{d \lambda}{d p}=\frac{t_{3}}{n} \frac{1}{c h^{2} \frac{t_{3}}{n}}, \frac{d B_{n}}{d p}=\frac{d B_{n}}{d \lambda} \frac{d \lambda}{d p}, \\
& \text { находим } \frac{d B_{n}}{d \lambda}=\frac{n}{t_{3}} c^{2} p \frac{t_{3}}{n} \frac{d B_{n}}{d p} . \text { Следовательно } \alpha_{k}=\frac{t_{3}}{n} \frac{1}{c^{2} p_{k} \frac{t_{3}}{n}} \beta_{k},
\end{aligned}
$$

где $\beta_{k}=2 \operatorname{res} Z=2 \operatorname{res} Z_{n}(\lambda), p=i_{\omega_{k}}$.

Учитывая, что рассматриваемые полюсы $Z_{n}(\lambda)$ в $p$ - плоскости удовлетворяют уравнению $\operatorname{tg} \omega \frac{t_{3}}{n}=\zeta_{k}, k=1,2, \ldots, n / 2$, находим время задержки

$$
t_{3}=\frac{n}{\omega n / 2} \operatorname{arctg} \zeta_{n / 2}
$$

Перейдём выражениях (2) и (4) к пределу при $n \rightarrow \infty$. В результате выраже- 
ние (2) перейдёт в (1), а время задержки

$$
t_{3}=\lim \frac{n}{\omega n / 2} \operatorname{arctg} \zeta_{n / 2} \cdot n \rightarrow \infty
$$

Поскольку последовательность $\left\{\zeta_{k}\right\}$ имеет предел, то

$$
t_{3}=\lim \operatorname{arctg} \zeta_{n / 2} \cdot \lim \frac{n}{\omega n / 2} \cdot n \rightarrow \infty
$$

Следовательно

$$
0<\lim \operatorname{arctg} \zeta_{n / 2} \leq \frac{\pi}{2}, n \rightarrow \infty
$$

откуда следует, что

$$
0<t_{3} \leq \pi \lim \frac{k}{\omega_{k}} k \rightarrow \infty
$$

Таким образом для диэлектрического слоя с конечной толщиной распределение резонансных частот в ограниченной области частот может быть любым. Чтобы поверхностному импедансу (1) соответствовал диэлектрический слой конечной толщины необходимо и достаточно, как видно из выражения (7), выполнить асимптотическое условие: величина предела, стоящего в правой части формулы (7) должна быть конечной величиной, то есть резонансные частоты должны подчиняться условию:

$$
0<\lim \frac{k}{\omega_{k}}<\infty k \rightarrow \infty
$$

Из выражения (7) вытекает, что если величина предела

$$
\lim \frac{k}{\omega_{k}}=0, \infty, k \rightarrow \infty
$$

то время задержки (толщина диэлектрического слоя покрытия[ 3,5 ]) равно соответственно нулю и бесконечности.Условие (8) является слишком общим и гарантирует существование слоя конечной толщины. Чтобы в конце слоя был помещён электрический экран на резонансные частоты необходимо наложить дополнительные ограничения. Для этого поверхностное сопротивление (1) при конечном верхнем пределе $\mathrm{n}$ разложим в непрерывную дробь:

$$
Z_{n}=\frac{1}{i \omega C_{1}+\frac{1}{i \omega L_{2}+\frac{1}{i \omega C_{3}+\frac{1}{i \omega L_{4}+\ldots+\frac{1}{i \omega L_{2 n}}}}}}
$$


Из данного выражения можно получить формулу для последнего элемента лестничной схемы $L_{2 n}$ :

$$
L_{2 n}=\frac{1}{4 \sum_{k=1}^{n} \frac{1}{\beta_{k} Q_{2 n}^{\prime}\left(\omega_{k}\right)^{2}}}, Q_{2 n}=\prod_{k=1}^{n}\left(1-\omega^{2} / \omega_{k}^{2}\right) \text {. }
$$

При $n \rightarrow \infty$ выражение (10) перейдёт в (1), а индуктивность $L_{2 n}$ - в индуктивность последнего элемента слоя. Так как в конце слоя должен находиться электрический экран (обшивка самолёта или ракеты), то индуктивность при $n \rightarrow \infty$ должна стремиться к нулю, то есть ряд ( $\beta_{k}$-ограниченные положительные числа [ 3 ])

$$
\sum_{k=1}^{\infty} 1 / Q^{\prime}\left(\omega_{k}\right)^{2}
$$

должен расходиться. Выражение для $\mathrm{Q}(\omega)$ определяется соответствующей формулой (11) при $n \rightarrow \infty$.

Таким образом для того чтобы существовал диэлектрический слой с электрическим экраном его резонансные частоты $\omega_{1}<\omega_{2}<\omega_{3}<\ldots$ должны удовлетворять двум условиям: должно быть соблюдено неравенство (8) и ряд (12) должен расходиться. Точно такие же ограничения накладываются и на частотные области поглощения радиомаскировочных покрытий. При этом резонансные частоты являются центральными частотами областей поглощения.Следуя изложенной выше логики можно сформулировать дополнительные ограничения, накладываемые на импеданс диэлектрических покрытий.Чтобы нерегулярный диэлектрический слой с конечным временем задержки не содержал сосредоточенных диэлектрических включений, необходимо и достаточно, чтобы

$$
\begin{gathered}
\sum_{k=1}^{\infty} \frac{\beta_{k}}{\omega_{k}^{2}}<\infty, \\
\sum_{k=1}^{\infty} \frac{1}{\beta_{k} \omega_{k}^{2}\left[Q^{\prime}\left(\omega_{k}\right)\right]^{2}}<\infty, Q(\omega)=\prod_{k=1}^{\infty}\left(1-\frac{\omega^{2}}{\omega_{k}^{2}}\right) .
\end{gathered}
$$

При этом к границе слоя может быть подключено сосредоточенное диэлектрическое включение.

Из данного результата следует ряд следствий.

Следствие 1. Еесли существует предел $\lim _{k \rightarrow \infty} \beta_{k}(\tau)$, то волновое сопротивление слоя 
Міжвідомчий науково-технічний збірник «Адаптивні системи автоматичного управління» № 1' (32) 2018

$$
Z_{B}(\tau)=\frac{t_{3}-\tau}{2} \lim _{k \rightarrow \infty} \beta_{k}(\tau), 0 \leq \tau<t_{3}
$$

В частности, данное следствие справедливо для плавно нерегулярных слоёв.

Следствие 2. Если диэлектрический слой не содержит сосредоточенных включений при $0 \leq \tau<t_{3}$, то при больших $k$

$$
\frac{1}{k^{1+\delta}} \leq \beta_{k}(\tau) \leq k^{1+\delta}, \delta>0 .
$$

Следствие 3. Если исключить требование (13), то слой может содержать сосредоточенные последовательные индуктивные диэлектрические включения. Если исключить требование (14), то слой может содержать параллельные ёмкостные включения.В приложениях часто высокоомные и низкоомные отрезки слоёв, имеющих малое время задержки в заданном диапазоне частот, приближенно заменяют сосредоточенными элементами L и C. При выполнении условий $(13,14)$ данная процедура оказывается справедливой и для слоёв, волновое сопротивления которых обращается в бесконечность или в нуль при некоторых $\tau$. При этом:

$$
L=\int_{\tau_{1}}^{\tau_{2}} Z_{B}(\tau) d \tau, C \int_{\tau_{1}}^{\tau_{2}} Z_{B}(\tau)^{-1} d t
$$

где $\left[\tau_{1}, \tau_{2}\right], \tau_{1}<\tau_{2}-$ участок линии внутри, которого $Z_{8}(\tau)$ обращается в бесконечность или в нуль. Величина $\tau_{2}-\tau_{1}$ должна быть достаточно малой.

Приведенные выше рассуждения в полной мере распространяться и на проводимость.

Следствие 4. Чтобы существовал диэлектрический слой с входным сопротивлением

$$
z=\frac{\beta_{0}}{p}+\sum_{k=1}^{\infty} \frac{\beta_{k} p}{\rho^{2}+\omega_{k}^{2}}
$$

не содержащая сосредоточенных элементов, с волновым сопротивлением $0<$ $Z_{8}(0)<\infty$ и набором резонансных частот $0<\omega_{1}<\omega_{2}<\ldots$ (спектром $\left.\left\{\omega_{k}\right\}\right)$, нагруженная на лестничную цепь $C_{H 1}, L_{H 2}$, состоящую из $m$ элементов, необходимо и достаточно выполнить требование (12) и условия

$$
\sum_{k=1}^{\infty} \frac{\omega_{k}^{2(m-1)}}{\left[M^{\prime}\left(\omega_{k}\right)\right]^{2}}<\infty, \sum_{k=1}^{\infty} \frac{\omega_{k}^{2 m}}{\left[M^{\prime}\left(\omega_{k}\right)\right]^{2}}=\infty, M(\omega)=\omega Q(\omega) .
$$

При этом слой имеет конечное время задержки $0<t_{3}<\infty$. Если рассматривать покрытие, для которого нуль не является резонансной частотой $\left(\beta_{0}=0\right)$, то в условиях (19) надо $M(\omega)$ заменить на $Q(\omega)$ и под цепью нагрузки понимать лестничную цепь , начинающуюся с последовательной индуктивности. 
Следствие 5. Если из последовательности $0<\omega_{1}<\omega_{2}<\ldots$ исключить число $\omega_{\eta} \neq 0$, то новая последовательность будет спектром слоя, нагруженного на лестничную цепь из $(m-2)$ элементов. Если окажется, что $(m-2) \leq 0$, то это означает, что спектр соответствует диэлектрическому покрытию с магнитной стенкой . Такой случай возникает, когда исходная последовательность $0<\omega_{1}<\omega_{2}<\ldots$ соответствует слою нагруженного на ёмкость или диэлектрическое включение типа последовательный контур. Данный вывод непосредственно следует из соотношения

$$
\begin{gathered}
\frac{1}{C_{H 1}}=4 \sum_{k=1}^{\infty} \frac{\beta_{0}^{2}}{\beta_{k}\left[M^{\prime}\left(\omega_{k}\right)\right]^{2}}+\beta_{0}, \frac{1}{C_{H 1} L_{H 2}}=4 C_{H 1} \sum_{k=1}^{\infty} \frac{\beta_{0}^{2} \omega_{k}^{2}}{\beta_{k}\left[M^{\prime}(\omega k)\right]^{2}}, \\
\frac{1}{C_{H 1} L_{H 2} C_{H 3}}=4 \beta_{0}^{2} \sum_{k=1}^{\infty} \frac{\omega_{k}^{2} C_{H 1} L_{H 2}-1}{\beta_{k}\left[M^{\prime}\left(\omega_{k}\right)\right]^{2}}
\end{gathered}
$$

Если к исходной последовательности $0<\omega_{1}<\omega_{2}<\ldots$ добавить новое число $\omega_{\eta} \neq \omega_{\mathrm{k}}$, то новая последовательность будет спектром слоя, нагруженного на цепь с $(m+2)$ элементами.

Если из набора частот $0<\omega_{1}<\omega_{2}<\ldots$ исключить произвольные $N$ чисел, отличных от нуля, и добавить другие $N$ чисел, отличных от нуля, то вновь образованная последовательность будет спектром слоя с нагрузкой того же типа и с прежним количеством элементов.

При рассмотрении диэлектрических слоёв, для которых нуль не является резонансной частотой все выводы остаются справедливыми. Если из спектра $0<\omega_{1}<\omega_{2}<\ldots$ исключить число нуль, то последовательность $\omega_{1}<\omega_{2}<\ldots$ является спектром слоя, нагруженного на лестничную цепь $L_{H 1}, C_{H 2}, L_{H 3}, \ldots$, состоящей из $(m-1)$ элементов. Если количество элементов получается отрицательным числом или равным нулю, то это означает, что слой имеет магнитную или электрическую стенку. Из сказанного следует, если числа $0<\omega_{1}<\omega_{2}<\ldots$ являются спектром слоя с магнитной стенкой, то последовательность $\omega_{1}<\omega_{2}<\ldots$ является спектром слоя с электрической стенкой, но не наоборот.

Пример. Пусть

$$
\omega_{0}=0, \omega_{k}=\frac{(2 k-1) \pi}{2 t_{3}}, k=1,2 \ldots,
$$

То есть данные числа являються резонансними частотами короткозамкнутой однородной линии с временем задержки $t_{3}$, к которым добавлено ещё одно число нуль. Чтобы определить тип нагрузки линии с данным спектром, находим

$$
M(\omega)=\omega \prod_{k=1}^{\infty}\left(1-\frac{\omega^{2} 4 t_{3}^{2}}{(2 \pi-1)^{2} \pi^{2}}\right)=\omega \cos \omega t_{3} .
$$


Следовательно,

$$
\begin{array}{r}
M^{\prime}(\omega)=\cos \omega t_{3}-\omega t_{3} \sin \omega t_{3} . \\
\text { При } \omega=\omega_{k} \quad\left[M^{\prime}\left(\omega_{k}\right)\right]^{2}=\frac{\pi^{2}}{4}(2 k-1)^{2} .
\end{array}
$$

Проверим выполнение условий (2.9). Пусть $m=1$. Тогда

$$
\sum_{k=1}^{\infty} \frac{1}{\left[M^{\prime}\left(\omega_{k}\right)\right]^{2}}=\frac{4}{\pi^{2}} \sum_{k=1}^{\infty} \frac{1}{(2 k-1)^{2}}=\frac{1}{2}, \quad \sum_{k=1}^{\infty} \frac{\omega_{k}^{2}}{\left[M^{\prime}\left(\omega_{k}\right)\right]^{2}}=\infty .
$$

Таким образом, числа $\omega_{k}$ образуют спектр диэлектрического покрытия с ёмкостной нагрузкой. Если к данной последовательности добавить ещё одно число, отличное от имеющихся, то новый числовой набор будет спектром слоя, нагруженного на лестничную цеп $C_{H 1}, L_{H 2}, C_{H 3}$.

\section{Выводы.}

В статье Теоретически обоснована возможность моделирования процесса эффективной радиолокации летательных аппаратов с искусственно сниженной ЕПР и новое решение научной проблемы, суть которой заключается в разработке нового принципа получения и использования радиолокационной информации о воздушной цели с искусственно пониженной эффективной площадью отражения на основе резонансной частотно - фазовой взаимосвязи СВЧ электромагнитного поля с кристаллической структурой радиопоглощающего покрытия.

\section{Список использованных источников}

1. В. В. Цветков, В. П. Демин, А. И. Куприянов. Радиоэлектронная борьба. Радиомаскировка и помехозащита. Вузовская книга - 2012.-240 с.

2. Коваль Ю. О., Милютченко І. О., Олейніков А. М., Шокало, В. М., Браїловський В. В., Бзовий Е. Г., Александров В. В. Основи теорії кіл, сигналів та процесів в системах технічного захисту інформації. НТМТ. - Харків.- 2011.- 544 с.

3. Гиллемин Е. А. Синтез пассивных цепей.-М.: Связь,1970.-720с.

4. Пархомей I. Р. Щодо можливості використання концентрованого резонансного електромагнітного поля. / І. Р. Пархомей//Зб. наук. пр. «Труди академії»., -: К. НАОУ, 2005p.-№ 61, c.102-108.

5. Пархомей І. Р. Методи підвищення ефективності локації цілей зі штучно зниженою ефективною площею віддзеркалення. / І. Р. Пархомей// Зб. наук.пр. «Труди академії»., -: К. HAOУ, 2005p. - № 66, c. 83-92. 